\title{
Dentate gyrus-specific knockdown of adult neurogenesis impairs spatial and object recognition memory in adult rats
}

\author{
Sebastian Jessberger, ${ }^{1,2,9}$ Robert E. Clark, ${ }^{3,4,9}$ Nicola J. Broadbent, ${ }^{4}$ \\ Gregory D. Clemenson Jr., ${ }^{1}$ Antonella Consiglio, ${ }^{1,5}$ D. Chichung Lie, ${ }^{1,6}$ \\ Larry R. Squire, $3,4,7,8,10$ and Fred H. Gage ${ }^{1,10}$ \\ ${ }^{1}$ Laboratory of Genetics, Salk Institute for Biological Studies, La Jolla, California 92037, USA; ${ }^{2}$ Institute of Cell Biology, Department \\ of Biology, ETH Zurich, 8093 Zurich, Switzerland; ${ }^{3}$ Veterans Affairs Medical Center, San Diego, California 92161, USA; \\ ${ }^{4}$ Department of Psychiatry, University of California, San Diego, California 92191, USA; ${ }^{5}$ Centre de Medicina Regenerativa, 08003 \\ Barcelona, Spain; ${ }^{6}$ HelmholtzZentrum München, German Research Center for Environmental Health, 85764 Munich, Germany; \\ ${ }^{7}$ Department of Neurosciences, University of California, San Diego, California 92161, USA; ${ }^{8}$ Department of Psychology, University \\ of California, San Diego, California 92161, USA
}

\begin{abstract}
New granule cells are born throughout life in the dentate gyrus of the hippocampal formation. Given the fundamental role of the hippocampus in processes underlying certain forms of learning and memory, it has been speculated that newborn granule cells contribute to cognition. However, previous strategies aiming to causally link newborn neurons with hippocampal function used ablation strategies that were not exclusive to the hippocampus or that were associated with substantial side effects, such as inflammation. We here used a lentiviral approach to specifically block neurogenesis in the dentate gyrus of adult male rats by inhibiting WNT signaling, which is critically involved in the generation of newborn neurons, using a dominant-negative WNT (dnWNT). We found a level-dependent effect of adult neurogenesis on the long-term retention of spatial memory in the water maze task, as rats with substantially reduced levels of newborn neurons showed less preference for the target zone in probe trials $>2$ wk after acquisition compared with control rats. Furthermore, animals with strongly reduced levels of neurogenesis were impaired in a hippocampus-dependent object recognition task. Social transmission of food preference, a behavioral test that also depends on hippocampal function, was not affected by knockdown of neurogenesis. Here we identified a role for newborn neurons in distinct aspects of hippocampal function that will set the ground to further elucidate, using experimental and computational strategies, the mechanism by which newborn neurons contribute to behavior.
\end{abstract}

[Supplemental material is available online at www.learnmem.org.]

Neural progenitors persist throughout life in the subgranular zone (SGZ) of the dentate gyrus and continuously give rise to new neurons (Zhao et al. 2008). Newborn granule cells pass through several developmental steps, from a dividing progenitor to a mature granule cell that becomes indistinguishable from granule cells born during embryonic development (Kempermann et al. 2004; Laplagne et al. 2006; Suh et al. 2007). After an activity-dependent selection process, newborn granule cells integrate into the preexisting dentate circuitry (van Praag et al. 2002; Kempermann et al. 2003; Tashiro et al. 2006). Interestingly, there is a critical period during which newborn neurons are more excitable than neurons that were born during embryogenesis or early postnatal (Schmidt-Hieber et al. 2004; Ge et al. 2007), suggesting that new neurons might not simply replace mature cells but that they have unique electrophysiological properties during early maturational stages that are functionally important in the context of adult neurogenesis.

The number of newborn neurons is not static but is dynamically regulated by a variety of factors (Ming and Song 2005).

\footnotetext{
${ }^{9}$ These authors contributed equally to this work.

${ }^{10}$ Corresponding authors.

E-mail gage@salk.edu; fax (858) 597-0824.

E-mail Isquire@ucsd.edu; fax (858) 552-7457.

Article is online at http://www.learnmem.org/cgi/doi/10.1101//m.1172609.
}

Manipulations to experimental animals that increase the number of newborn granule cells, such as physical activity or environmental enrichment, are associated with improved cognitive performance, whereas aging and stress impair both neurogenesis and hippocampus-dependent behavior (Kempermann et al. 1997; Gould et al. 1999; van Praag et al. 2005; Montaron et al. 2006). Thus, new neurons might be important for hippocampal function. Indeed, one previous report suggested that newborn granule cells become preferentially integrated into networks coding for spatial memory (Kee et al. 2007). To date, a number of studies have tried to link hippocampal neurogenesis and behavior by using a variety of methods in mice and rats to block adult neurogenesis, including cytostatic drugs, brain irradiation, and genetic ablation of dividing progenitors throughout the adult brain (e.g., Shors et al. 2001; Santarelli et al. 2003; Saxe et al. 2006; Winocur et al. 2006). These studies have obtained variable and also partially contradictory results, perhaps related to the method of knockdown, which species is used, or the nature of the behavioral task. There is also a technical challenge in studying the functional significance of adult-born granule cells, because only a small fraction of the total neuronal cell population is newborn at a given time, and one would want to selectively manipulate this population.

We have previously shown that WNT signaling plays a pivotal role in the generation of newborn neurons in the adult dentate 
gyrus (Lie et al. 2005). Inhibition of WNT signaling within the dentate gyrus using a lentivirus expressing dominant-negative WNT (dnWNT) almost completely abolished the formation of newborn neurons without affecting progenitor division in any other brain area (Lie et al. 2005). We utilized this strategy to block neurogenesis in the hippocampus of adult rats. To this end, we stereotactically injected into the dentate gyrus of rats with either control virus or virus expressing dnWNT to inhibit the generation of new neurons. Rats with normal levels of neurogenesis and rats with mildly or strongly reduced levels of neurogenesis were challenged with three hippocampus-dependent behavioral tasks. By use of this approach, we identified a level-dependent role for newborn neurons in the long-term retention of spatial memory and in hippocampus-dependent forms of object recognition memory.

\section{Materials and Methods}

\section{Animals and stereotactic injections}

All animal procedures were performed in accordance with protocols approved by the animal care use committee of the Salk Institute for Biological Studies and the University of California, San Diego. Animals were purchased from Harlan (San Diego, CA) and kept under a constant 12-h light/dark cycle and with access to food and water ad libitum. A total of 72 male, 7- to 8-wk-old Sprague Dawley rats (175-200 g) were used. Experimental rats $(n=$ 36 ) were injected in three independent cohorts ( $n=12$ per cohort) with lentivirus-expressing CMV-driven dnWNT followed by an internal ribosomal entry site (IRES)-green fluorescent protein (GFP) from the same vector (see below). Control rats $(n=36)$ were also injected in three cohorts with a lentivirus-expressing CMVdriven GFP. For all surgeries, animals were deeply anesthetized with a ketamine/xylazine mixture. A 30-gauge needle with point style 4 (Hamilton) was used for all injections. The first two cohorts received two intrahippocampal injections of $1.5 \mu \mathrm{L}$ viral suspension (see below) at $-3.2 \mathrm{a} / \mathrm{p}, \pm 1.2 \mathrm{~m} / \mathrm{l},-4.1 \mathrm{~d} / \mathrm{v}$ and $-5 \mathrm{a} / \mathrm{p}, \pm 2.9$ $\mathrm{m} / \mathrm{l},-3.8 \mathrm{~d} / \mathrm{v}$ (all values from bregma) per side. In the third cohort, we increased the number of injections to nine per dentate area $(0.3$ $\mu \mathrm{L}$ viral suspension per injection site). Coordinates for rats from the third cohort were $(\mathrm{a} / \mathrm{p}, \mathrm{m} / \mathrm{l}, \mathrm{d} / \mathrm{v}$ from Bregma) $-2.4, \pm 1,-4.1$; $-3.2, \pm 1.2,-4.1 ;-4, \pm 2,-3.7 ;-4.8, \pm 3,-3.8 ;-5.4, \pm 3.4,-4$; $-5.4, \pm 4.4,-7.2 ;-6, \pm 4,-4.2 ;-6, \pm 4,-7.4 ;-6.3, \pm 4.4,-4$. All injections were performed manually with an injection rate of $0.1-$ $0.3 \mu \mathrm{L} / \mathrm{min}$.

\section{Lentiviral vector production}

We used the previously described lentiviral vectors (Lie et al. 2005): The transfer vector CSC.cPPT.hCMV.eGFP.Wpre (expressing GFP) or CSC.cPPT.hCMV.dnWNT.iresGFP.Wpre (expressing dnWNT and GFP from the same vector) and the packaging plasmids pMDLg/pRRE, pRSV-Rev and pMD2.VSV-G. LV stocks were produced by calcium phosphate transfection into 293T cells, as previously described (Follenzi and Naldini 2002; Tiscornia et al. 2006). Briefly, supernatants were collected, passed through a 0.22 $\mu \mathrm{m}$ filter, and purified by ultracentrifugation as described. Expression titers were estimated on 293T cells by limiting dilution. Concentrated vector expression titer ranged from $1 \times 10^{9}$ and 1 $\times 10^{10}$ transducing units $(\mathrm{TU}) / \mathrm{mL}$ for each vector. All viral stocks used for injections were diluted to $1 \times 10^{9} \mathrm{TU} / \mathrm{mL}$.

\section{Histology}

Animals were deeply anesthetized and transcardially perfused with $0.9 \% \mathrm{NaCl}$ followed by $4 \%$ paraformaldehyde (PFA) in $0.1 \mathrm{M}$ phosphate buffer ( $\mathrm{pH}$ 7.4). Brains were fixed for an additional $24 \mathrm{~h}$ in $4 \%$ PFA and then transferred into 30\% sucrose. Fortymicrometer-thick coronal sections were cut using a sliding micro- tome. Immunohistochemistry was performed as described previously (Jessberger and Kempermann 2003). Every 12th section was stained using antibodies against DCX (1:250, Santa Cruz Biotechnology) and GFP (1:500, Molecular Probes, Millipore). All sections were also stained with 4',6-diamidino-2-phenylindol (DAPI, 1:5000, Sigma). DCX-positive cells were counted throughout the rostrocaudal extent of the granule cell layer (GCL) of the dentate gyrus on both sides using a $20 \times$ objective and an upright epifluorescence microscope (Eclipse E800; Nikon). The derived numbers were then multiplied by 12 to obtain the total number of DCX-positive cells per animal. To analyze the coverage of the GCL by viral expression, we used the trace function of the StereoInvestigator software (MicroBrightField) controlling an epifluorescence microscope (Nikon Eclipse E600). The total area of the GCL was measured by the DAPI signal; lentivirus-transduced areas were measured by GFP expression using every twelfth section throughout the rostrocaudal extent of the hippocampus.

\section{Behavioral tasks}

Behavioral testing was started 8-9 wk after injection of lentiviral vectors. Each group was trained first on the water maze task and then later given retention tests. Each rat was also given a test of object recognition memory (novel object recognition [NOR]; with three different delay intervals), and cohorts 1 and 3 were given a test of social transmission of food preference (STFP). Accordingly, each rat was given acquisition training on the water maze task and then a retention probe trial that was presented $2 \mathrm{wk}, 4 \mathrm{wk}$, or $8 \mathrm{wk}$ after the last day of acquisition training. Each rat was also tested on the object recognition task with delay intervals of $1 \mathrm{~min}, 3 \mathrm{~h}$, and $4 \mathrm{wk}$. Rats tested on the STFP task were given a 48-h retention interval. Finally, each rat was then given acquisition training on a new water maze in a different room or reversal training on the same water maze (cohorts 1 and 2). The order of the object recognition test and the STFP test were counterbalanced, as were the delay intervals of the object recognition memory test (see Supplemental Fig. 1).

\section{Water maze}

The benchmark test of spatial memory in the rodent is the water maze (Martin and Clark 2007). Lesions of the hippocampus that encompass as little as 30\%-50\% of total hippocampal volume cause severe impairment on this task (Moser et al. 1993, 1995; Broadbent et al. 2004).

\section{Apparatus}

Testing was conducted in the Morris water maze (diameter $1.8 \mathrm{~m}$ ) with an "Atlantis platform" (diameter $12.7 \mathrm{~cm}$ ) (Spooner et al. 1994), which could be raised or lowered remotely during a trial. The platform was located in the center of the northeast quadrant of the pool throughout testing. The water was rendered opaque by the addition of powdered milk, and four $30-\mathrm{W}$ spotlights pointed at the ceiling illuminated the room. The water was maintained at $23^{\circ} \mathrm{C}$. The testing room contained a number of constant, salient visual cues (posters, objects, and equipment). A video camera was mounted on the ceiling directly above the pool and was used, in conjunction with a video tracking system (San Diego Instruments), to record the swim path of each rat.

\section{Spatial training}

Each rat received one training session each day for $7 \mathrm{~d}$. Each daily session began with a single reinforced probe trial, followed by four training trials. For the probe trials, the platform was lowered so that it was inaccessible, and the rat was placed in the water facing the pool wall at one of four start points (north, south, east, or west). The start point used was counterbalanced across trials for all 
animals. Upon release into the water, the rat was allowed to swim for $60 \mathrm{sec}$, at which point the platform was raised to within $1.5 \mathrm{~cm}$ of the water surface. An additional $60 \mathrm{sec}$ were then allowed for the rat to locate the platform and escape from the water. After escaping, the rat remained on the platform for $30 \mathrm{sec}$ before being removed. If the rat failed to escape, it was guided to the platform and remained there for $30 \mathrm{sec}$.

The performance measure was the percentage of time that animals spent in a $30-\mathrm{cm}$-diameter circular zone surrounding the platform location (4\% of the total pool surface area, chance $4 \%$ ). Previous work has indicated that basing water maze performance on the time spent in a small circle around the platform location provides a more sensitive measure of memory than the standard measure of time spent in the training quadrant (Moser et al. 1993). Whereas the quadrant measure indicates whether animals remember the general location of the platform, the small circle measure assesses how well animals remember its exact location.

\section{Retention probes}

Following acquisition, subsets of rats were given a 60 -sec retention probe trial after a 2-, 4-, or 8-wk retention delay.

\section{New spatial learning}

Following the retention probes, each rat was tested for new spatial learning ability by training with a new platform location (cohorts 1 and 2) or on a new water maze (cohort 3 ). This training protocol was identical to the initial acquisition protocol.

\section{Novel object recognition (NOR)}

The NOR task takes advantage of a rodent's spontaneous preference to explore novel objects relative to familiar objects. This test is the benchmark test of recognition memory in the rodent and is dependent on the integrity of the hippocampus (for review, see Squire et al. 2007).

\section{Apparatus}

NOR was tested in an opaque plastic chamber $(35 \mathrm{~cm} \times 41.5 \mathrm{~cm} \times 50$ $\mathrm{cm}$ high). A video camera mounted on the wall directly above the chamber was used to record the testing session for offline analysis. Overhead fluorescent lighting illuminated the testing area.

\section{Procedure}

A single NOR trial consists of a familiarization phase (encoding) followed by a prescribed delay interval and then a test phase (retrieval). During the familiarization phase, the rat was allowed to explore and become familiar with two identical objects placed side by side in the chamber. Following the familiarization phase, delay intervals of $1 \mathrm{~min}, 3 \mathrm{~h}$, and $1 \mathrm{mo}$ intervened before the test phase (the order of delay intervals was counterbalanced across rats). For the 1-min and 3-h delay intervals, a single 15-min familiarization phase was presented before the test phase. For the 1-mo delay, each animal was given 15 min of familiarization phase exposure (using the identical pair of objects) each day for four consecutive days.

During the test phase, the rat was allowed to explore two objects placed side by side (one novel object and a third, identical copy of an object from the familiarization phase). We scored object exploration (nose within $2 \mathrm{~cm}$ of object and vibrissae moving) (see Clark et al. 2000) until the rat accumulated $30 \mathrm{sec}$ of contact time with the objects. The dependent measure was the percentage of time that a rat spent exploring the novel object during the $30 \mathrm{sec}$ of object exploration. Which object was novel and the left/right position of the novel object was counterbalanced within each group, and new pairs of objects were used for each NOR test. The experimenter was blind to the group membership of the rats during testing and offline data analysis.

\section{Social transmission of food preference}

In STFP, a "demonstrator" rat that has recently eaten a scented food is exposed to a "subject" rat. Subsequently, the subject rat expresses its memory for the odor through a preference for the same scented food. During this social interaction, the subject rat makes an association between the food odor and constituents of the demonstrator's breath (Galef Jr. et al. 1983). Subsequently, when the subject rat is presented with a choice between two odorous foods, the subject rat expresses a memory for the acquired association by choosing the same food odor that was present on the demonstrator's breath. This task is sensitive to hippocampal dysfunction (Winocur 1990; Bunsey and Eichenbaum 1995; Alvarez et al. 2001; Winocur et al. 2001; Clark et al. 2002; Ross and Eichenbaum 2006).

\section{Procedure}

The task consisted of three distinct phases.

\section{Phase I}

Demonstrator rats were first accustomed for $4 \mathrm{~d}$ to a routine of $23 \mathrm{~h}$ of food deprivation, followed by $1 \mathrm{~h}$ of feeding. During the feeding, 40-50 g of meal chow was available in a glass jar attached to the floor of the demonstrator's cage. Following the feeding session, the food was weighed, and the amount of food eaten was recorded.

\section{Phase II (interaction)}

Demonstrator rats were next exposed to flavored meal chow during each day's 1 -h feeding session ( $1 \%$ cinnamon, i.e., $1 \mathrm{~g}$ cinnamon/100 g meal chow, or $2 \%$ cocoa, i.e., $1 \mathrm{~g}$ cocoa $/ 50 \mathrm{~g}$ meal chow). Each demonstrator was fed only one of the two flavors. After $4 \mathrm{~d}$, when demonstrator rats were always eating at least $5 \mathrm{~g}$ of the flavored food during the daily feeding session, the demonstrator rat was allowed to interact with a subject rat for $30 \mathrm{~min}$. Specifically, the demonstrator rat was placed in the home cage of a subject rat but separated from the subject rat by a cylindershaped wire screen. The subject rat was food deprived for $1 \mathrm{~h}$ before the interaction, and no food or water was available to either rat during the interaction. The subject and demonstrator rats were always unfamiliar with each other.

\section{Phase III (test)}

Following a 48-h delay, the subject rat was food deprived for $8 \mathrm{~h}$ and then presented in its home cage with a choice of a novel food and the food that the demonstrator rat had consumed before interacting with the subject rat (familiar food). Two jars were attached to the floor of the subject rat's cage and remained in place for $2 \mathrm{~h}$. One jar was filled with meal chow flavored with $1 \%$ cinnamon, and the other jar was filled with meal chow flavored with $2 \%$ cocoa. The familiar flavor (cinnamon or cocoa) was counterbalanced across all conditions, as was the right or left location of the familiar food. At the end of the 2-h feeding period, the jars were weighed and the amount of food eaten from each jar was recorded. The percentage preference for the familiar food was calculated as $[\mathrm{F} /(\mathrm{F}+\mathrm{N}) \times 100]$, where $\mathrm{F}$ is the amount of familiar food consumed and $\mathrm{N}$ is the amount of novel food consumed.

\section{Results}

\section{Lentivirus-mediated knockdown of neurogenesis}

Dividing progenitor cells are present in many areas of the adult brain. However, new neurons are generated only in two distinct areas, the subventricular zone (SVZ) of the lateral ventricles and the SGZ of the dentate gyrus, suggesting the existence of local cues that enable the formation and maturation of newborn neurons. 

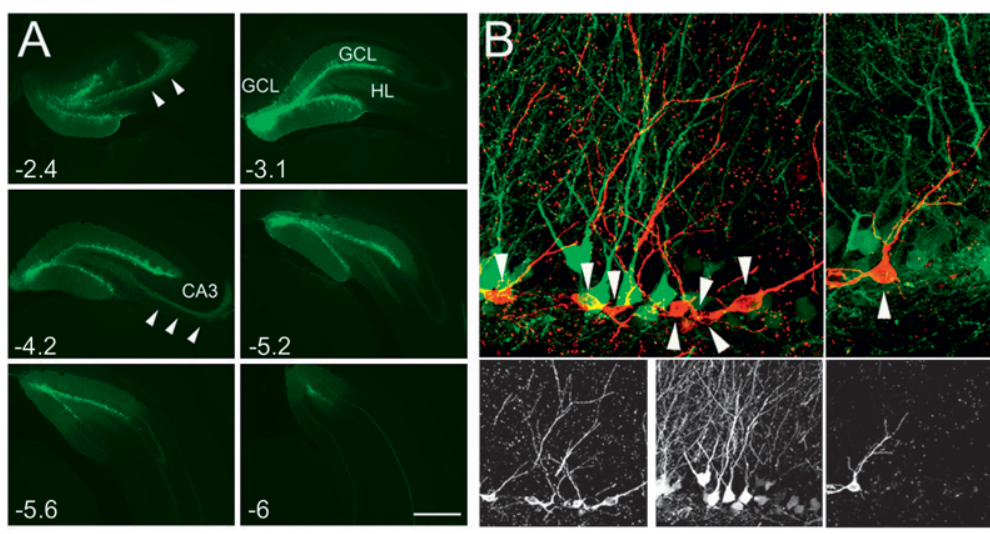

Figure 1. Lentiviral expression of dnWNT reduces levels of adult hippocampal neurogenesis. $(A)$ Stereotactic injection of lentiviral vectors effectively targets the adult dentate area. Shown is an example of an animal that had received two intrahippocampal injections of control lentivirus expressing GFP (green). Note that GFP expression is largely confined to the dentate area, spanning from anterior parts of the dentate gyrus to posterior areas (numbers in the left lower corner are approximate distances from bregma). Arrowheads point toward mossy fibers extending from lentivirus-transduced granule cells. (B) Lentiviral expression decreases the number of newborn neurons expressing DCX (red, arrowheads in $B$ ). Shown are areas that were transduced (expressing GFP, green) with a control lentivirus (left panels) or dnWNT-expressing lentivirus (right panels). Single channels for GFP and DCX are shown in small panels below multichannel images. Scale bars, $300 \mu \mathrm{m}(A), 10 \mu \mathrm{m}(B)$. GCL indicates granule cell layer; $\mathrm{HL}$, hilus; and CA3, cornu ammonis area 3.

We stereotactically injected three independent cohorts of adult male rats with control lentivirus expressing GFP or with lentivirus expressing dnWNT into the dentate area (Fig. 1A). Levels of neurogenesis were measured by quantifying the number of DCXexpressing cells. DCX is a microtubule-associated protein that is transiently expressed in the course of adult neurogenesis. The number of DCX-expressing cells directly correlates with conventional measures of adult neurogenesis, such as BrdU/NeuN colabeling, making it a suitable endogenous marker of adult neurogenesis under conditions where repeated intraperitoneal injections of BrdU might cause stress-related behavioral effects. Confirming our previous results, injection of dnWNT substantially decreased the number of DCX-expressing cells in the SGZ (GFP $=11,655 \pm$ SEM 465 vs. dnWNT $=6628 \pm 818$ SEM; $t_{(70)}=5.3, P<0001$ ) (Figs. $1 \mathrm{~B}, 2)$, and this reduction was apparent and reliable in both the dorsal and ventral portions of the hippocampus.

\section{Experimental groups}

There was considerable variability in the degree of neurogenesis knockdown (Fig. 2). For the experimental group, DCX cell counts were expressed as a percentage of the mean DCX counts for the control group. We noted that several subjects had higher DCX counts than the control mean and many more had only modest levels of DCX reduction. Accordingly, at 35\% of control level we split the experimental group into a high- and low-knockdown group (HIGH-KD and LOW-KD, respectively). The mean for the HIGH-KD group (mean DCX counts $=2988 \pm 226$ SEM) was $26 \pm$ $1.9 \%$ of controls, and the mean for the LOW-KD group (mean DCX counts $=9886 \pm 1085$ SEM) was $85 \pm 9.3 \%$ of controls. In this way, we tested the function of newborn neurons in hippocampus-dependent behavior in a dose-dependent manner.

\section{Spatial learning and memory}

\section{Acquisition}

Figure $3 \mathrm{~A}$ shows probe trial performance during the $7 \mathrm{~d}$ of acquisition training. A repeated-measures ANOVA revealed a main effect of training day $\left(F_{(6,396)}=60.34, P<0.0001\right)$ but no effect of group $\left(F_{(2,396)}=0.13, P>0.1\right)$. The same findings were obtained

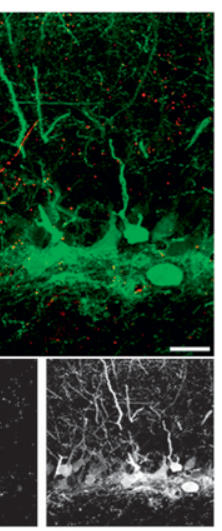

when we measured the time spent in the training quadrant (main effect of training day, $F_{(6,396)}=68.30, P<0.0001 ;$ no group effect, $\left.F_{(2,396)}=0.71, P>0.1\right)$ and when we analyzed the latency and distance the animals swam to find the platform (data not shown). Thus, the three groups acquired the water maze task equally well.

\section{Retention}

Three delay intervals were tested: 2 -wk (control $n=6$; HIGH-KD $n=1$; LOW-KD $n=5$ ), 4-wk (control $n=18$; HIGH-KD $n=$ 14; LOW-KD $n=4$ ), or 8-wk retention delay (control $n=12$; HIGH-KD $n=2$; LOW-KD $n=10$ ). The average delay interval based on group membership was control $=35 \mathrm{~d}$, HIGH-KD $=31 \mathrm{~d}$, and LOW-KD = $39 \mathrm{~d}$. The retention performance of the control group across the three delay intervals was not different $\left(\right.$ ANOVA; $F_{(2,33)}=1.3, P=0.30 ; F_{(2,33)}=$ $0.4, P=0.70$ for circle and quadrant measures, respectively). Thus, to evaluate retention, we combined performance scores across all three retention delay intervals (Fig. 3B). For the small training zone, the HIGH-KD group was impaired relative to the LOW-KD group $\left(t_{(34)}=2.2, P<0.05\right)$ and marginally impaired relative to the control group $\left(t_{(51)}=1.92, P=0.06\right)$. The LOW-KD and control

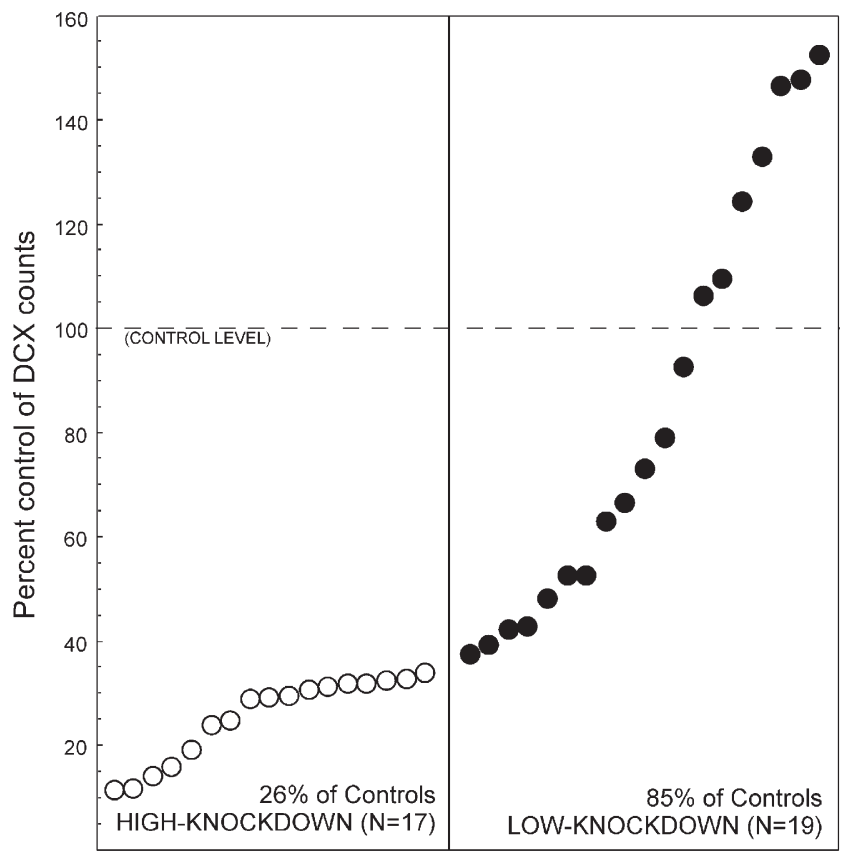

Figure 2. The distribution of hippocampal neurogenesis knockdown. Circles represent a single animal's DCX cell count expressed as a percentage of the mean DCX count for the control group. There was considerable variability in the degree of neurogenesis knockdown. Accordingly, we split the experimental group into high- and low-knockdown groups. Animals with knockdowns less than $35 \%$ of controls were designated HIGH-KD. The mean group knockdown for this group was $26 \%$ of controls. Animals with knockdowns that were greater than $35 \%$ of controls were designated LOW-KD. The mean group knockdown for this group was $85 \%$ of controls. 


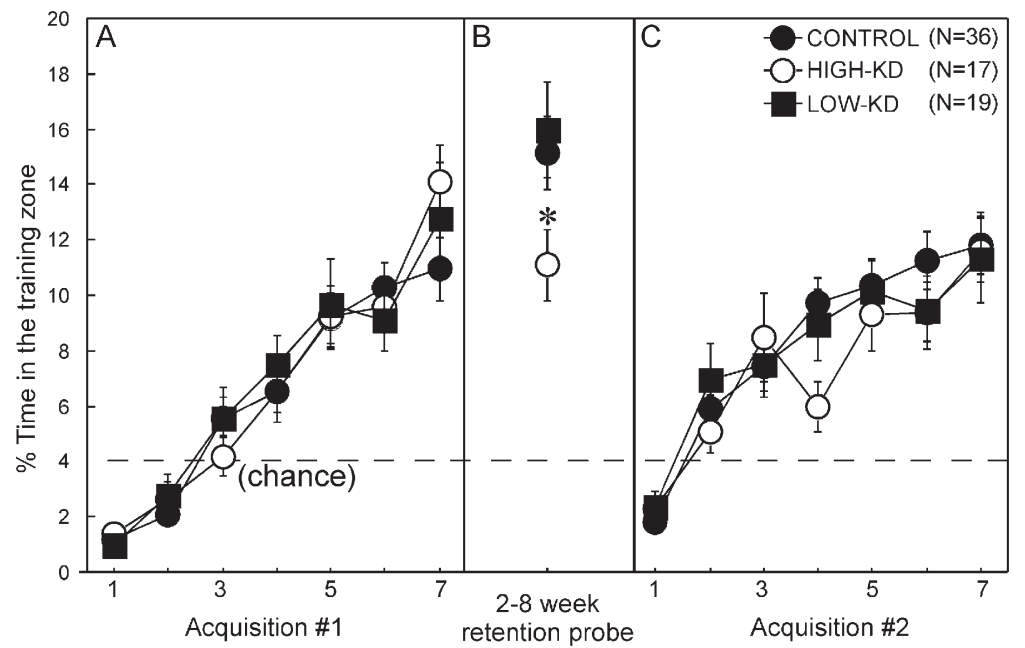

Figure 3. Water maze probe trial performance across $7 \mathrm{~d}$ of acquisition training $(A)$, performance on a retention probe trial given after a 2 - to 8 -wk retention interval $(B)$, and probe trial performance across $7 \mathrm{~d}$ of acquisition training for a new platform location (C). Black circles represent the control group $(n=$ 36 ), white circles represent the high-knockdown group $(n=17)$, and black squares represent the lowknockdown group $(n=19)$. Asterisk indicates impaired performance of the high-knockdown group relative to the low-knockdown group $(P<0.05)$.

groups performed similarly $(P>0.1)$. For the training quadrant measure, the HIGH-KD group was marginally impaired relative to the LOW-KD group (control $=51.2 \pm 2.7 \%$; HIGH-KD $=47.3 \pm$ $3.1 \%$; LOW-KD $\left.=58.8 \pm 3.9 \% ; t_{(34)}=1.92, P=0.06\right)$. The HIGH-KD and control groups performed similarly $\left(t_{(51)}=0.97, P>0.1\right)$. All groups performed above chance on both the zone and quadrant measures (all $t s>5.5$, all $P \mathrm{~s}<0.001$ ). This finding indicates that although the HIGH-KD group was impaired, all groups were exhibiting substantial memory retention.

\section{New spatial learning}

A repeated-measures ANOVA revealed a main effect of training day $\left(F_{(6,342)}=36.71, P<0.0001\right)$ but no effect of group $\left(F_{(2,342)}=0.02\right.$, $P>0.1$ ) when a circle analysis was used. The same findings were obtained when we measured the time spent in the training quadrant [main effect of training day $\left(F_{(6,342)}=63.85, P<\right.$ $0.0001)$, no group effect $\left.\left(F_{(2,342)}=0.01, P>0.1\right)\right]$. These findings indicate that the three groups acquired the new water maze task equally well.

\section{Novel object recognition}

\section{Retention test}

For the 1-min delay interval, all groups performed above chance (all $t \mathrm{~s}>4.21$, all $P \mathrm{~s}<0.01$ ) and there were no group differences ( $t \mathrm{~s}<$ $1.03, P s>0.10)$. This indicates that all of the groups exhibited substantial memory retention at this delay (Fig. 4). For the 3-h delay interval, the HIGH-KD group failed to perform better than chance levels $\left(t_{(16)}=1.71, P>0.1\right)$ and also performed more poorly than the LOW-KD group $\left(t_{(34)}=2.28, P<0.05\right)$. The LOW-KD and control groups performed similarly $(t s<1.61, P>0.10)$, and both groups performed above chance ( $t \mathrm{~s}>6.12, P \mathrm{~s}<0.01$ ). For the 1 -mo delay interval, no significant group differences emerged $(t s<0.96$, $P>0.10)$. Nonetheless, the LOW-KD and control groups preferentially explored the novel object as their preference was above chance ( $t \mathrm{~s}>2.52, P \mathrm{~s}<0.05$ ), indicating some preserved memory retention. In contrast, the HIGH-KD group failed to demonstrate preserved memory retention as they did not spend significantly more time exploring the novel object and did not perform above chance $\left(t_{(16)}=1.76, P=0.10\right)$, indicating a substantial difference in the performance of the HIGH-KD groups compared with LOW-KD and control groups (Fig. 4).

\section{Social transmission of food preference}

\section{Retention test}

For the 48-h delay interval, all groups performed above chance (all $t s>3.25$, all $P \mathrm{~s}<0.01)$ and there were no group differences $(t s<1.50, P \mathrm{~s}>0.10)$. This indicates that all three groups showed substantial and equal memory retention (Fig. 5).

\section{Discussion}

We have used a lentivirus-based strategy to block neurogenesis in the dentate gyrus. Using this approach, we identified a role for newborn granule cells in the long-term retention of spatial memory and object recognition memory, whereas a third hippocampus-dependent task was unaffected.

In the spatial memory task (water maze), acquisition progressed normally in all groups. Yet when retention tests took place $2-8 \mathrm{wk}$ later, the group with the most knockdown of neurogenesis (HIGH-KD) performed more poorly than the LOW-KD and control groups. When the groups were trained in a new water maze, all groups learned equally well. This finding suggests that memory ability was not simply declining across time but rather was specific to the demanding long-retention tests.

For the object recognition task, all three groups performed above chance and equally well at a retention delay of $1 \mathrm{~min}$. Yet at the more demanding delay intervals of $3 \mathrm{~h}$ and $4 \mathrm{wk}$, group differences emerged. At the 3-h delay, the HIGH-KD group failed to perform above chance and performed more poorly that the LOW-KD group. At the 4-wk delay, the HIGH-KD group was the only group that failed to perform above chance, whereas the LOW$\mathrm{KD}$ and control groups spent significantly more time exploring the novel object even at long retention intervals. These findings can be contrasted with the normal performance of the HIGH-KD group on the STFP task.

With respect to spatial memory, results from previous studies are not in agreement. Whereas some studies found no impairment

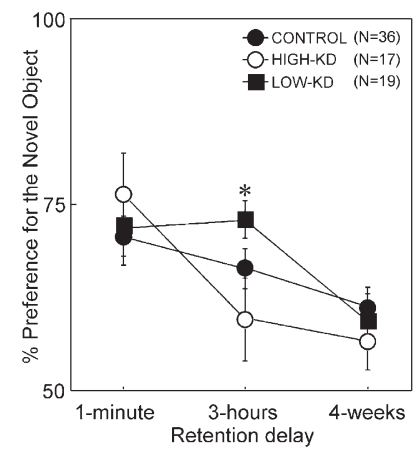

Figure 4. Percentage of preference for the novel object across the 1-min, 3-h, and 4-wk delay intervals. Black circles represent the control group $(n=36)$, white circles represent the high-knockdown group $(n=$ $17)$, and black squares represent the low-knockdown group $(n=19)$. Asterisk indicates impaired performance of the high-knockdown group relative to the low-knockdown group $(P<0.05)$. 


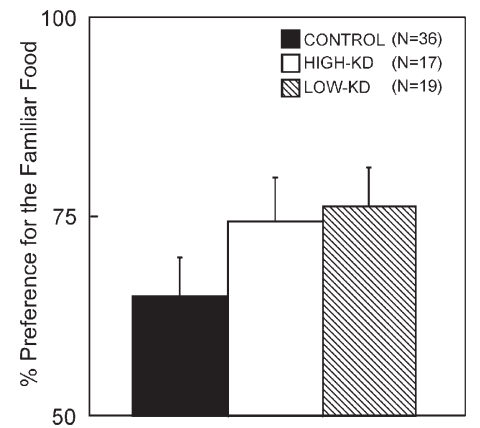

Figure 5. Percentage of preference for the familiar food following a 48-h delay. Black bar represents the control group $(n=36)$, white bar represents the high-knockdown group $(n=17)$, and the striped bar represents the low-knockdown group $(n=19)$. All groups performed above chance and equally well.

in the water maze (using irradiation and MAM) (Shors et al. 2002; Meshi et al. 2006), others described substantial deficits in longterm retention (using irradiation [Snyder et al. 2005] or diphtheria toxin-based ablation of new neurons [Imayoshi et al. 2008]) or even impairment in the acquisition phase of spatial learning (using genetic ablation of TLX or transgenesis-mediated ablation of newborn cells) (Dupret et al. 2008; Zhang et al. 2008). The explanation for these discrepancies remains unclear, but it seems reasonable to suppose that the differences are related to the method used to block neurogenesis. In addition, species differences and training protocols could be contributing factors. Our results, which are based on a dentate-specific block of newborn neurons, identify a role for new neurons in the long-term expression of spatial memory in adult rats. It is noteworthy that the phenotype observed here is not consistent with a full dentate gyrus lesion. Complete lesions of the dentate gyrus produce substantial impairment in water maze acquisition (Xavier et al. 1999 ) as do lesions that disconnect CA3 from CA1 (Brun et al. 2002; but see Nakashiba et al. 2008). Further, because our method leaves the dentate gyrus as a whole intact, future studies will have to determine if depletion of neurogenesis results in impaired performance in tasks that are specifically designed to assess the function of the dentate gyrus, such as tests of pattern separation (e.g., Gilbert et al. 2001).

We also found a significant impairment in a hippocampusdependent form of object recognition memory. Consistent with this observation, there is evidence that aging, which is associated with a dramatic decline in the number of newborn neurons, is also associated with a decline in object recognition in rodents and humans (Schacter et al. 1992; Scali et al. 1997). Furthermore, another recent study showed that the improved object recognition performance associated with environmental enrichment is dependent on the generation of new neurons (Bruel-Jungerman et al. 2005). Thus, the data presented here indicate that adult neurogenesis is important for object recognition memory (i.e., when the delay interval is $3 \mathrm{~h}$ or longer).

Earlier studies aiming to understand the contribution of newborn neurons to context memory are also controversial. For example, classical conditioning of context fear has been reported to be impaired after blocking neurogenesis (Saxe et al. 2006; Winocur et al. 2006; Imayoshi et al. 2008; Wojtowicz et al. 2008), whereas contextual fear conditioning has also been reported to be intact after blocking hippocampal neurogenesis (Dupret et al. 2008; Zhang et al. 2008).

The finding that new granule cells are born throughout life in the mammalian dentate gyrus has triggered considerable speculation about how neurogenesis might contribute to hippocampus- dependent behavior, and a number of studies have been carried out to explore this issue. The first studies aiming to link neurogenesis causally with behavior used cytostatic drugs such as methyl-azoxy-methanol (MAM), which blocks cell proliferation (Shors et al. 2001, 2002). However, this strategy caused potentially severe side effects due to inhibition of cell proliferation in the whole animal, complicating the interpretation of the results (Dupret et al. 2005). More recent studies used transgenic mice expressing thymidine kinase under the control of promoters that are active in neural stem cells, such as glial fibrillary acidic protein (GFAP). This manipulation led to a substantial reduction of neurogenesis after Ganciclovir injection (Garcia et al. 2004; Saxe et al. 2006, 2007). However, the behavioral phenotype was difficult to interpret because blocking of the dividing precursors occurred not only in the dentate gyrus but also in a second neurogenic area, the SVZ of the lateral ventricles (and potentially in other organs, such as intestines in the case of GFAP) (Bush et al. 1998). A similar expression in the SVZ occurred after the conditional deletion of a key gene for neural stem cell maintenance/ differentiation, the transcription factor TLX (Zhang et al. 2008). Improved spatial restriction was subsequently obtained by more focal hippocampal irradiation, which resulted in robust and persisting depletion of dividing progenitors in the dentate area (Santarelli et al. 2003; Saxe et al. 2006). However, irradiation might also affect post-mitotic neurons and cause a substantial inflammatory response (Monje et al. 2003).

We have tried to overcome the technical concerns of these earlier studies by using a lentivirus-based strategy that specifically targets the dentate gyrus. We had previously shown that WNT signaling is critically involved in hippocampal neurogenesis, and in our current study, we used multiple bilateral injections of a lentivirus expressing a dnWNT to inhibit functional WNT signaling (Lie et al. 2005). With this approach, we were able to (1) restrict genetic manipulation to the dentate gyrus; (2) block neurogenesis exclusively in the dentate gyrus and not in other neurogenic areas; (3) initiate the block at a specific time in adulthood; (4) use wild-type animals, avoiding potential genetic background effects; and (5) use rats in which hippocampal function is well-characterized based on extensive work with lesions.

Our approach also has potential complications. There is evidence that WNT signaling might be implicated in synaptic plasticity of mature dentate granule cells (Chen et al. 2006; see also Beaumont et al. 2007). Thus, one should consider the possibility that the lentivirus-based expression of a dnWNT interfered with synaptic plasticity in post-mitotic granule cells. However, previous work has indicated that blocking hippocampal synaptic plasticity (by blocking NMDA receptors and LTP) does not impair spatial memory retention in the water maze but does impair acquisition (Steele and Morris 1999; Day and Morris 2001; Bast et al. 2005). Because our findings are the reverse (intact acquisition, impaired retention) of this pattern of impairment, it is unlikely that impaired synaptic plasticity of post-mitotic granule cells was a contributing factor.

A number of hypotheses have been developed to explain how new neurons might contribute to hippocampal function (Schinder and Gage 2004; Becker 2005; Aimone et al. 2006; Kempermann 2008). Newborn neurons represent only a small cell population within the adult dentate gyrus. How might this relatively small number of cells influence the function of hippocampal circuitry? Interestingly, at the age of $\sim 3-5 \mathrm{wk}$, new neurons differ substantially from mature granule cells. One feature of young newborn neurons that distinguishes them from mature granule cells is that they exhibit a reduced threshold for induction of long-term potentiation (LTP) (Wang et al. 2000; Schmidt-Hieber et al. 2004; Ge et al. 2007). Accordingly, it has been proposed that 
the new neurons that are young when events occur have a specialized role in encoding and storage and in temporally relating one event to another (Aimone et al. 2006).

The use of established hippocampus-dependent behavioral tasks is a logical starting point for understanding the contribution of adult neurogenesis in the hippocampus to behavior. Tests such as the water maze are sensitive to conventional hippocampal lesions and even conventional lesions targeting the dentate gyrus (Xavier et al. 1999). However, these hippocampus-dependent tests yielded only a modest impairment in the water maze task when neurogenesis was blocked. Furthermore, new neurons appear not to be involved in all hippocampus-dependent forms of learning and memory, as no impairment occurred for STFP. The challenge will be to design tasks that depend on the specific contribution provided by newborn neurons in the context of current ideas about hippocampal function. In this regard, it is also important to note that our data suggest that neurogenesis is involved in some but not all forms of hippocampus-dependent learning memory, because experimental animals were impaired in retention of spatial and object recognition memory yet performance on the STFP task was not affected. The design of tasks that are sensitive to the status of neurogenesis will likely be guided by the known effects of hippocampal lesions, by the particular characteristics of newborn cells, and by the computational models that make predictions about how newborn cells can influence hippocampal function.

\section{Acknowledgments}

This work was supported by grants from the NCCR Neural Plasticity and Repair and MaxnetAging (to S.J.), the Medical Research Service of the Department of Veterans Affairs, the National Institute of Mental Health, the Metropolitan Life Foundation, National Institute of Aging grant (P50 AG05131), the National Science Foundation, and the James S. McDonnell Foundation, NINDS (NS050217), as well as a National Alliance for Research on Schizophrenia and Depression Effie Beeman Investigator Award. We thank Laura Entwistle, Daniel Guadarrama, Stephane Gaskin, and Brittany Masatsugu for their assistance. We also thank Mary Lynn Gage for editorial comments.

\section{References}

Aimone, J.B., Wiles, J., and Gage, F.H. 2006. Potential role for adult neurogenesis in the encoding of time in new memories. Nat. Neurosci. 9: 723-727.

Alvarez, P., Lipton, P.A., Melrose, R., and Eichenbaum, H. 2001. Differential effects of damage within the hippocampal region on memory for a natural, nonspatial odor-odor association. Learn. Mem. 8: 79-86.

Bast, T., da Silva, B.M., and Morris, R.G. 2005. Distinct contributions of hippocampal NMDA and AMPA receptors to encoding and retrieval of one-trial place memory. I. Neurosci. 25: 5845-5856.

Beaumont, V., Thompson, S.A., Choudhry, F., Nuthall, H., Glantschnig, H., Lipfert, L., David, G.R., Swain, C.J., McAllister, G., and Munoz-Sanjuan, I. 2007. Evidence for an enhancement of excitatory transmission in adult CNS by Wnt signaling pathway modulation. Mol. Cell. Neurosci. 35: $513-524$.

Becker, S. 2005. A computational principle for hippocampal learning and neurogenesis. Hippocampus 15: 722-738.

Broadbent, N.J., Squire, L.R., and Clark, R.E. 2004. Spatial memory, recognition memory, and the hippocampus. Proc. Natl. Acad. Sci. 101: 14515-14520.

Bruel-Jungerman, E., Laroche, S., and Rampon, C. 2005. New neurons in the dentate gyrus are involved in the expression of enhanced long-term memory following environmental enrichment. Eur. J. Neurosci. 21: 513-521.

Brun, V.H., Otnass, M.K., Molden, S., Steffenach, H.A., Witter, M.P., Moser M.B., and Moser, E.I. 2002. Place cells and place recognition maintained by direct entorhinal-hippocampal circuitry. Science 296: 2243-2246.

Bunsey, M. and Eichenbaum, H. 1995. Selective damage to the hippocampal region blocks long-term retention of a natural and nonspatial stimulus-stimulus association. Hippocampus 5: 546-556.

Bush, T.G., Savidge, T.C., Freeman, T.C., Cox, H.J., Campbell, E.A., Mucke, L., Johnson, M.H., and Sofroniew, M.V. 1998. Fulminant jejuno-ileitis following ablation of enteric glia in adult transgenic mice. Cell 93: 189-201.

Chen, J., Park, C.S., and Tang, S.J. 2006. Activity-dependent synaptic Wnt release regulates hippocampal long term potentiation. J. Biol. Chem. 281: $11910-11916$.

Clark, R.E., Zola, S.M., and Squire, L.R. 2000. Impaired recognition memory in rats after damage to the hippocampus. J. Neurosci. 20: $8853-8860$.

Clark, R.E., Broadbent, N.J., Zola, S.M., and Squire, L.R. 2002. Anterograde amnesia and temporally graded retrograde amnesia for a nonspatial memory task after lesions of hippocampus and subiculum. J. Neurosci. 22: 4663-4669.

Day, M. and Morris, R.G. 2001. Memory consolidation and NMDA receptors: Discrepancy between genetic and pharmacological approaches. Science 293: 755. doi: 10.1126/science.293.5531.755a.

Dupret, D., Montaron, M.F., Drapeau, E., Aurousseau, C., Le Moal, M., Piazza, P.V., and Abrous, D.N. 2005. Methylazoxymethanol acetate does not fully block cell genesis in the young and aged dentate gyrus. Eur. J. Neurosci. 22: 778-783.

Dupret, D., Revest, J.M., Koehl, M., Ichas, F., De Giorgi, F., Costet, P., Abrous, D.N., and Piazza, P.V. 2008. Spatial relational memory requires hippocampal adult neurogenesis. PLoS One 3: e1959. doi: 10.1371/ journal.pone.0001959.

Follenzi, A. and Naldini, L. 2002. HIV-based vectors. Preparation and use. Methods Mol. Med. 69: 259-274.

Galef Jr., B.G., Wigmore, S.W., and Kennett, D.J. 1983. A failure to find socially mediated taste aversion learning in Norway rats ( $R$. norvegicus). J. Comp. Psychol. 97: 358-363.

Garcia, A.D., Doan, N.B., Imura, T., Bush, T.G., and Sofroniew, M.V. 2004. GFAP-expressing progenitors are the principal source of constitutive neurogenesis in adult mouse forebrain. Nat. Neurosci. 7: $1233-1241$.

Ge, S., Yang, C.H., Hsu, K.S., Ming, G.L., and Song, H. 2007. A critical period for enhanced synaptic plasticity in newly generated neurons of the adult brain. Neuron 54: 559-566.

Gilbert, P.E., Kesner, R.P., and Lee, I. 2001. Dissociating hippocampal subregions: Double dissociation between dentate gyrus and CA1. Hippocampus 11: 626-636.

Gould, E., Beylin, A., Tanapat, P., Reeves, A., and Shors, T.J. 1999. Learning enhances adult neurogenesis in the hippocampal formation. Nat. Neurosci. 2: 260-265.

Imayoshi, I., Sakamoto, M., Ohtsuka, T., Takao, K., Miyakawa, T., Yamaguchi, M., Mori, K., Ikeda, T., Itohara, S., and Kageyama, R. 2008. Roles of continuous neurogenesis in the structural and functional integrity of the adult forebrain. Nat Neurosci. 11: 1153-1161.

Jessberger, S. and Kempermann, G. 2003. Adult-born hippocampal neurons mature into activity-dependent responsiveness. Eur. J. Neurosci. 18: 2707-2712.

Kee, N., Teixeira, C.M., Wang, A.H., and Frankland, P.W. 2007. Preferential incorporation of adult-generated granule cells into spatial memory networks in the dentate gyrus. Nat. Neurosci. 10: 355-362.

Kempermann, G. 2008. The neurogenic reserve hypothesis: What is adult hippocampal neurogenesis good for? Trends Neurosci. 31: 163-169.

Kempermann, G., Kuhn, H.G., and Gage, F.H. 1997. More hippocampal neurons in adult mice living in an enriched environment. Nature 386: 493-495.

Kempermann, G., Gast, D., Kronenberg, G., Yamaguchi, M., and Gage, F.H. 2003. Early determination and long-term persistence of adult-generated new neurons in the hippocampus of mice. Development 130: 391-399.

Kempermann, G., Jessberger, S., Steiner, B., and Kronenberg, G. 2004. Milestones of neuronal development in the adult hippocampus. Trends Neurosci. 27: 447-452.

Laplagne, D.A., Esposito, M.S., Piatti, V.C., Morgenstern, N.A., Zhao, C., van Praag, H., Gage, F.H., and Schinder, A.F. 2006. Functional convergence of neurons generated in the developing and adult hippocampus. PLoS Biol. 4: e409. doi: 10.1371/journal.pbio.0040409.

Lie, D.C., Colamarino, S.A., Song, H.J., Desire, L., Mira, H., Consiglio, A., Lein, E.S., Jessberger, S., Lansford, H., Dearie, A.R. , et al. 2005. Wnt signalling regulates adult hippocampal neurogenesis. Nature 437: 1370-1375.

Martin, S.J. and Clark, R.E. 2007. The rodent hippocampus and spatial memory: From synapses to systems. Cell. Mol. Life Sci. 64: 401-431.

Meshi, D., Drew, M.R., Saxe, M., Ansorge, M.S., David, D., Santarelli, L., Malapani, C., Moore, H., and Hen, R. 2006. Hippocampal neurogenesis is not required for behavioral effects of environmental enrichment. Nat. Neurosci. 9: 729-731.

Ming, G.L. and Song, H. 2005. Adult neurogenesis in the mammalian central nervous system. Annu. Rev. Neurosci. 28: 223-250.

Monje, M.L., Toda, H., and Palmer, T.D. 2003. Inflammatory blockade restores adult hippocampal neurogenesis. Science 302: 1760-1765.

Montaron, M.F., Drapeau, E., Dupret, D., Kitchener, P., Aurousseau, C., Le Moal, M., Piazza, P.V., and Abrous, D.N. 2006. Lifelong corticosterone 
level determines age-related decline in neurogenesis and memory. Neurobiol. Aging 27: 645-654.

Moser, E., Moser, M.B., and Andersen, P. 1993. Spatial learning impairment parallels the magnitude of dorsal hippocampal lesions, but is hardly present following ventral lesions. J. Neurosci. 13: 3916-3925.

Moser, M.B., Moser, E.I., Forrest, E., Andersen, P., and Morris, R.G. 1995. Spatial learning with a minislab in the dorsal hippocampus. Proc. Natl. Acad. Sci. 92: 9697-9701.

Nakashiba, T., Young, J.Z., McHugh, T.J., Buhl, D.L., and Tonegawa, S. 2008 Transgenic inhibition of synaptic transmission reveals role of CA3 output in hippocampal learning. Science 319: 1260-1264.

Ross, R.S. and Eichenbaum, H. 2006. Dynamics of hippocampal and cortical activation during consolidation of a nonspatial memory. J. Neurosci. 26: $4852-4859$.

Santarelli, L., Saxe, M., Gross, C., Surget, A., Battaglia, F., Dulawa, S., Weisstaub, N., Lee, J., Duman, R., Arancio, O. , et al. 2003. Requirement of hippocampal neurogenesis for the behavioral effects of antidepressants. Science 301: 805-809.

Saxe, M.D., Battaglia, F., Wang, J.W., Malleret, G., David, D.J., Monckton, J.E., Garcia, A.D., Sofroniew, M.V., Kandel, E.R., Santarelli, L. , et al. 2006. Ablation of hippocampal neurogenesis impairs contextual fear conditioning and synaptic plasticity in the dentate gyrus. Proc. Natl. Acad. Sci. 103: 17501-17506.

Saxe, M.D., Malleret, G., Vronskaya, S., Mendez, I., Garcia, A.D., Sofroniew, M.V., Kandel, E.R., and Hen, R. 2007. Paradoxical influence of hippocampal neurogenesis on working memory. Proc. Natl. Acad. Sci. 104: $4642-4646$.

Scali, C., Giovannini, M.G., Prosperi, C., Bartolini, L., and Pepeu, G. 1997. Tacrine administration enhances extracellular acetylcholine in vivo and restores the cognitive impairment in aged rats. Pharmacol. Res. 36: 463-469.

Schacter, D.L., Cooper, L.A., and Valdiserri, M. 1992. Implicit and explicit memory for novel visual objects in older and younger adults. Psychol. Aging 7: 299-308.

Schinder, A.F. and Gage, F.H. 2004. A hypothesis about the role of adult neurogenesis in hippocampal function. Physiology (Bethesda) 19: 253-261.

Schmidt-Hieber, C., Jonas, P., and Bischofberger, J. 2004. Enhanced synaptic plasticity in newly generated granule cells of the adult hippocampus. Nature 429: 184-187.

Shors, T.J., Miesegaes, G., Beylin, A., Zhao, M., Rydel, T., and Gould, E. 2001. Neurogenesis in the adult is involved in the formation of trace memories. Nature 410: $372-376$.

Shors, T.J., Townsend, D.A., Zhao, M., Kozorovitskiy, Y., and Gould, E. 2002 Neurogenesis may relate to some but not all types of hippocampaldependent learning. Hippocampus 12: 578-584.

Snyder, J.S., Hong, N.S., McDonald, R.J., and Wojtowicz, J.M. 2005. A role for adult neurogenesis in spatial long-term memory. Neuroscience 130: 843-852.
Spooner, R.I., Thomson, A., Hall, J., Morris, R.G., and Salter, S.H. 1994. The Atlantis platform: A new design and further developments of Buresova's on-demand platform for the water maze. Learn. Mem. 1: 203-211.

Squire, L.R., Wixted, J.T., and Clark, R.E. 2007. Recognition memory and the medial temporal lobe: A new perspective. Nat. Rev. Neurosci. 8: 872-883.

Steele, R.J. and Morris, R.G. 1999. Delay-dependent impairment of a matching-to-place task with chronic and intrahippocampal infusion of the NMDA-antagonist D-AP5. Hippocampus 9: 118-136.

Suh, H.K., Consiglio, A., Ray, J., Sawai, T., D'Amour, K.A., and Gage, F.H. 2007. In vivo fate analysis reveals the multipotent and self-renewal capacities of Sox2 $2^{+}$neural stem cells in the adult hippocampus. Cell Stem Cell 1: 515-528.

Tashiro, A., Sandler, V.M., Toni, N., Zhao, C., and Gage, F.H. 2006. NMDAreceptor-mediated, cell-specific integration of new neurons in adult dentate gyrus. Nature 442: 929-933.

Tiscornia, G., Singer, O., and Verma, I.M. 2006. Production and purification of lentiviral vectors. Nat. Protocols 1: 241-245.

van Praag, H., Schinder, A.F., Christie, B.R., Toni, N., Palmer, T.D., and Gage, F.H. 2002. Functional neurogenesis in the adult hippocampus. Nature 415: $1030-1034$.

van Praag, H., Shubert, T., Zhao, C., and Gage, F.H. 2005. Exercise enhances learning and hippocampal neurogenesis in aged mice. J. Neurosci. 25: 8680-8685.

Wang, S., Scott, B.W., and Wojtowicz, J.M. 2000. Heterogenous properties of dentate granule neurons in the adult rat. J. Neurobiol. 42: 248-257.

Winocur, G. 1990. Anterograde and retrograde amnesia in rats with dorsal hippocampal or dorsomedial thalamic lesions. Behav. Brain Res. 38: $145-154$.

Winocur, G., McDonald, R.M., and Moscovitch, M. 2001. Anterograde and retrograde amnesia in rats with large hippocampal lesions. Hippocampus 11: $18-26$.

Winocur, G., Wojtowicz, J.M., Sekeres, M., Snyder, J.S., and Wang, S. 2006. Inhibition of neurogenesis interferes with hippocampus-dependent memory function. Hippocampus 16: 296-304.

Wojtowicz, J.M., Askew, M.L., and Winocur, G. 2008. The effects of running and of inhibiting adult neurogenesis on learning and memory in rats. Eur. J. Neurosci. 27: 1494-1502.

Xavier, G.F., Oliveira-Filho, F.J., and Santos, A.M. 1999. Dentate gyrusselective colchicine lesion and disruption of performance in spatial tasks: Difficulties in "place strategy" because of a lack of flexibility in the use of environmental cues? Hippocampus 9: 668-681.

Zhang, C.L., Zou, Y., He, W., Gage, F.H., and Evans, R.M. 2008. A role for adult TLX-positive neural stem cells in learning and behaviour. Nature 451: $1004-1007$.

Zhao, C., Deng, W., and Gage, F.H. 2008. Mechanisms and functional implications of adult neurogenesis. Cell 132: 645-660.

Received July 31, 2008; accepted in revised form December 12, 2008. 


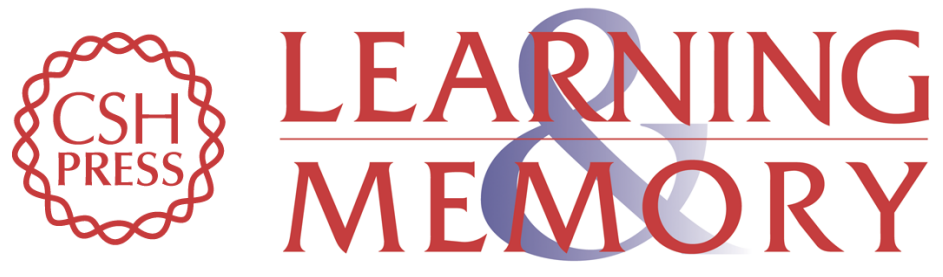

\section{Dentate gyrus-specific knockdown of adult neurogenesis impairs spatial and object recognition memory in adult rats}

Sebastian Jessberger, Robert E. Clark, Nicola J. Broadbent, et al.

Learn. Mem. 2009, 16:

Access the most recent version at doi:10.1101//m.1172609 Supplemental
Material http://learnmem.cshlp.org/content/suppl/2009/01/30/16.2.147.DC1

References This article cites 66 articles, 18 of which can be accessed free at: http://learnmem.cshlp.org/content/16/2/147.full.html\#ref-list-1

License

Email Alerting Receive free email alerts when new articles cite this article - sign up in the box at the Service top right corner of the article or click here. 